\title{
Historia y epistemología de la función derivada
}

\author{
History and epistemology of the derivative function
}

\author{
Eliseo Ramírez Rincón
}

Profesor de matemáticas Universidades U.D.C.A y Libre. elmatematis@gmail.com . Estudiante de doctorado en Educación UPN.

\section{Resumen}

La historia y la epistemología de la función derivada como objeto del cálculo diferencial dan cuenta de la complejidad y de los vaivenes que en veinte siglos ha sufrido ésta, hasta adquirir el estatus de función derivada. El trabajo de cientos de seres humanos dedicados a su estudio, en distintas épocas y culturas, han hecho aportes que han permitido los cambios y el refinamiento de las ideas matemáticas de la función derivada para convertirla en un objeto (puro, aplicado y a enseñar), muy potente. Es tal la importancia de este objeto matemático que permite resolver problemas de las matemáticas, de las ciencias naturales, sociales y humanas.

El aspecto central de este trabajo es presentar algunas consideraciones abordando la complejidad de la función derivada como objeto a enseñar y como objeto enseñado, desde la epistemología estándar y echar una mirada al mismo objeto desde otras epistemologías.

\section{Palabras clave}

Derivada, función, historia, epistemología, didáctica, cognición

\section{Abstract}

The history and epistemology of derivative function as a differential calculus object, talk about the complexity and the fluctuations that it has undergone to acquire derivative function status during the last twenty centuries. Many people have studied and have worked on it at different cultures and times, too. They have done contributions that have allowed to make changes on the derivative function, in order this to be as we know it today, because it has been being developed and shaped itself along this time to turn it into a very potent (formal, applied and for teaching) object. It is so the importance of this mathematical object that it allows to solve problems from mathematics itself, and of natural, social and human sciences.

The central aspect of this lecture is to present some considerations around the complexity of derivative function as a teaching object and also as a taught object, from standard epistemology as well as to throw a glance on the same object from a non standard epistemology

\section{Keywords}

Derivative, function, history, epistemology, didactic, cognition 


\section{Introducción}

Entre las complejidades de la función derivada se encuentra su lenguaje: la simbología, las representaciones (internas-externas), los contextos de aplicación, las interpretaciones (sintácticas y semánticas) y la relación enseñanza-aprendizaje. De acuerdo con lo anterior es posible estudiar la complejidad de la función derivada en cuatro dimensiones: Histórica, epistemológica, cognitiva y didáctica. Se aclara que para algunos investigadores como Artigue (1995), solo son tres las dimensiones, porque se considera como una de ellas, la historia y la epistemología. Para este trabajo la historia muestra el camino a la epistemología que no necesariamente es único.

El interés de esta propuesta es evidenciar desde la historia el camino de la epistemología y en ese sentido, presentar otras posibles epistemologías de la función derivada, que favorecen el proceso de enseñanza aprendizaje de éste, porque la epistemología usual del objeto puro no necesariamente responde a las necesidades e implicaciones de las relaciones entre el objeto a enseñar, el objeto enseñado, la cultura, los contextos de uso y demás relaciones que subyacen a su enseñanza.

La Didáctica de la Matemática (Matemática Educativa- Educación Matemática) ${ }^{1}$ como ciencia emergente intenta responder entre otras a las preguntas: ¿Qué enseñar? Y ¿Cómo enseñar? En el caso de este trabajo estudia las relaciones establecidas entre la función derivada como objeto matemático, la función derivada como objeto a enseñar y la función derivada como objeto enseñado, sin descuidar el entorno local y global como aspecto cultural y social en el que se propone, así como tampoco los usos de ésta y su lenguaje.

\section{Desarrollo}

Desde 1823, cuando Cauchy definió el objeto función derivada, se han propuesto algunos trabajos respecto a la misma, sin embargo la mayoría de ellos retoma la definición de Cauchy para su elaboración; es el caso de los trabajos propuestos sobre la función derivada por Caratheodory (teoría de funciones de variable compleja, 1954), Fréchet (diferencial total, 1963) y Gâteaux (derivada direccional, 1925). Alrededor de estos trabajos se han hecho propuestas sobre la didáctica de la función derivada, pero centradas en el pensamiento avanzado del cálculo, aspecto que no beneficia a los estudiantes de: último año del colegio, de los primeros semestres de ingeniería o de otras carreras que estudian el cálculo; entre otras razones por la complejidad del objeto, por el nivel con el que pasan del colegio a la universidad en cuanto a las ideas previas de función. Estas propuestas que se han presentado están en el análisis estándar, porque corresponden a la epistemología de Cauchy, en la que el límite como objeto matemático es el que permite estudiar a la función derivada y más aún son muy novedosas y ricas en recursos y estrategias en el nivel de pensamiento matemático avanzado, situación que no favorece el proceso de aprendizaje de un estudiante que apenas está incursionando en el cálculo. De otra parte se encuentran las que se han hecho en el análisis estándar, pero con otra epistemología, como la propuesta hecha por Cantoral (1995), al retomar el trabajo de Lagrange sobre series, evitando así el paso al límite para abordar la función derivada. Esta propuesta de Cantoral está en un nivel de pensamiento básico del cálculo y eso permite que sea una alternativa en el proceso de enseñanza aprendizaje de la función derivada con estudiantes de los primeros semestres. En el mismo sentido se puede construir una propuesta en el

\footnotetext{
${ }^{1}$ La Didáctica de la Matemática (Europa continental) y La Educación Matemática (descendencia Anglosajona), hacen referencia a lo mismo; su diferencia es puramente geográfica. Cantoral 2000
} 
análisis no estándar con los hiperreales de Robinson² (análisis no-estándar), como una extensión de los reales. Con este enfoque se disminuye el peso del rigor matemático asumido por el lenguaje de la función derivada en el análisis estándar, acercando a los estudiantes "primíparos" a los fundamentos del cálculo desde la intuición, antes que del rigor matemático, dado que en el proceso natural del ser humano, éste primero aprende a hablar y luego a escribir y no al contrario. La intuición matemática fue muy importante desde los griegos hasta el siglo XVIII, cuando se logra el rigor matemático actual, inclusive Newton (fluxiones) y Leibniz (infinitésimos) de alguna manera lo usaron para desarrollar sus respectivos trabajos. A partir del rigor matemático alcanzado en el s. XVIII, la intuición matemática deja de considerarse importante e inclusive se desprecia en el ambiente matemático del objeto puro, pero la Didáctica de la Matemática debe dar cuenta del aspecto cognitivo, social y cultural en el que se desenvuelve el objeto y en ese sentido el razonamiento intuitivo es fundamental.

La intuición como razonamiento matemático es muy importante en el análisis no estándar, en éste se encuentra el conjunto de los hiperreales, los cuales contienen a los números infinitésimos e infinitos que no tienen cabida en los reales (análisis estándar), además en este nuevo conjunto se cumplen las propiedades de los números reales y su estructura es más intuitiva que rigurosa, se disminuye la pesada formulación matemática de la función derivada, por ejemplo la siguiente expresión permite mostrar las diferencias entre el análisis estándar y el no estándar, para determinar la continuidad ${ }^{3}$ de una función en un punto con abscisa $\boldsymbol{x}$.

$>$ Expresión clásica (análisis estándar):

$$
\begin{array}{r}
\forall \boldsymbol{x}, \forall \varepsilon>\mathbf{0}, \exists \delta>\mathbf{0}\left(\left(\left|\boldsymbol{x}-\boldsymbol{x}_{1}\right|<\boldsymbol{\delta} \Rightarrow\left|\mathbf{f}(\mathbf{x})-\mathbf{f}\left(\mathbf{x}_{1}\right)\right|<\varepsilon\right)\right) \\
\text { Expresión en análisis no estándar: } \forall \boldsymbol{x}\left(\left(\boldsymbol{x} \cong \boldsymbol{x}_{1} \Rightarrow \mathbf{f}(\mathbf{x}) \cong \mathbf{f}\left(\mathbf{x}_{1}\right)\right)\right)
\end{array}
$$

La expresión no estándar, es más intuitiva y práctica.

En general, los números hiperreales permiten suprimir muchos cuantificadores.

Acosta y Delgado (1994), se basaron en el trabajo que sobre la función derivada en los reales realizó Kuhn (1991), a partir de lo hecho por Caratheodory sobre la función derivada de variable compleja. Acosta y Delgado a partir de la idea de Kuhn propusieron la definición para funciones de $R^{n}$ en $R^{m}$, realizando algunas demostraciones y resolviendo algunos ejercicios propuestos, donde muestran como calcular derivadas utilizando la definición de Caratheodory. Además, establecen la equivalencia entre las definiciones de derivada dadas por Fréchet (diferencial total) y Caratheodory. Otra de las definiciones de diferencial es la conocida con el nombre de derivada de Gâteaux o derivada direccional. Se ha demostrado que una función diferenciable según Fréchet es diferenciable según Gâteaux, pero no lo es la afirmación contraria, por lo tanto, debido a la equivalencia entre la derivada de Caratheodory y la de Fréchet, toda función diferenciable según Caratheodory también es diferenciable según Gâteaux.

Estas propuestas, son muy importantes en el desarrollo, construcción y enseñanza del objeto matemático, pero dado su lenguaje riguroso y su compleja red en la que están incrustadas, como propuestas en la enseñanza de la función derivada los estudiantes requieren no sólo de las nociones básicas de la función derivada, sino también conocimientos de la compleja estructura matemática de ésta, es decir estas propuestas pueden ser exitosas en el desarrollo de pensamiento matemático avanzado.

\footnotetext{
${ }^{2}$ Abraham Robinson, nacionalizado en USA, matemático nacido en la actual Polonia, en la década del 70 propuso los hiperreales, como extensión del conjunto de los reales.

${ }^{3}$ La continuidad de la función derivada $\left(f^{\prime}(x)\right)$ en un punto $(x, y)$ permite determinar si la función $f$, es diferenciable en ese punto.
} 


\section{Síntesis histórica de la función derivada}

Desde los griegos, se plantearon cuatro problemas fundamentales que al ser resueltos en el s XVI-XVII, dieron vida a la función derivada, fueron ellos: El de la velocidad, el de la recta tangente, el de área bajo una curva y el de máximos y mínimos. Entre los trabajos destacados en la cultura griega, respecto a los procesos de variación se encuentran los de: Zenón de Elea 450 a.C., de la escuela Eleática, para quien el movimiento era imposible y consideraba que el espacio y el tiempo eran infinitamente divisibles. De él son famosas sus paradojas: La del movimiento, la de Aquiles, la de la flecha y la del tiempo. Luego está la escuela de los atomistas: Leucipio, Demócrito y Jenofonte, s. V y s IV a.C., quienes se preocuparon por atacar el idealismo de la escuela Eleática, centrándose en el otro extremo, el materialismo. Para esta escuela el movimiento correspondía a la interacción de los átomos y de alguna forma concibieron el movimiento como una relación del espacio y el tiempo. En el s. IV a. C aparece Eudoxio, considerado el padre de la astronomía, por lo tanto en su trabajo el movimiento era muy importante. En 370 a. C. logra plasmar su trabajo escrito sobre el método de exhausión, el cual era un método riguroso y esencialmente geométrico de hallar el área bajo una curva a través de polígonos inscritos y circunscritos, logra por este método hallar el área de un círculo. Este método posteriormente fue utilizado por Arquímedes (287-212 a. C.), considerado por algunos como uno de los tres matemáticos más brillantes de la historia, junto con Newton y Gauss, trabajó en matemática pura y aplicada, continuó con el método de exhausión y logró avances significativos en áreas bajo curvas, demostró por series el área de una región de parábola y otras regiones, trabajó en el movimiento y al igual que sus antecesores la intuición fue de vital importancia para su trabajo.

Es evidente que los anteriores nombres hacen parte de los matemáticos griegos que lograron avances significativos en la geometría y en la aritmética, también lo es la dificultad que tuvieron para trabajar con el infinito y el hecho de que si los matemáticos griegos y filósofos como Platón y Aristóteles hubiesen seguido el camino de Arquímedes y no solo el de Euclides, el desarrollo de las matemáticas se hubiese adelantado varios siglos.

Hasta el siglo XVI, los matemáticos retoman el trabajo de los griegos respecto a los procesos de variación para resolver problemas que se planteaban desde la mecánica, en ese sentido se retoman los trabajos de Eudoxio y de Arquímedes sobre el método de exhausión para hallar áreas bajo curvas. Aparecen matemáticos como: Luca Valerio (1552-1618), Galileo (1564, 1642), Kepler (1571-1630), Huygens (1596-1695), Descartes (1596-1650), Cavalieri (1598-1647), Fermat (1601-1665), Roberval (16021675), Torricelli (1608-1647), Wallis (1616-1703), Pascal (1623-1662), Hudde (16281704) y Barrow (1630-1677). En este período el rigor matemático cambia respecto del usado por los griegos (Geométrico), se hace necesario buscar nuevas formas de demostrar los procesos matemáticos distintos a los de la geometría y del álgebra, se estudian las relaciones del movimiento, áreas bajo curvas, recta tangente y máximos y mínimos como procesos de variación, en este período la intuición como razonamiento matemático también era muy importante. Se encuentran diferencias en el rigor utilizado por los matemáticos de esta época y en ese sentido por ejemplo se destacan los trabajos de Fermat, Descartes Galileo y de Barrow. En general los trabajos de estos matemáticos en el cálculo, antecedieron al de Newton (1643-1727) en su teoría de fluxiones y al de Leibniz (1646-1716) en la teoría infinitesimal, ambos por caminos distintos con lenguajes también diferentes lograron darle piso a lo que hoy se conoce como cálculo diferencial e integral. Tanto Newton como Leibniz, usaron los infinitésimos y los infinitos e intentaron dejarlos de lado por las críticas que algunos 
pensadores como Berkeley (1685-1753) les hicieron, este hecho marca otra etapa más en el avance del rigor matemático el cual tuvo que esperar hasta los trabajos de Cauchy (1789-1957) a quien se le atribuye el rigor actual de las matemáticas, la definición y la definición de función derivada entre otros, Dedekind (1831-1916) sobre cortaduras y Cantor (1845-1918) sobre conjuntos. Son los trabajos de estos tres matemáticos los que finalmente permiten a las matemáticas y en particular al cálculo establecerse como un dominio matemático distinto al del álgebra, al de la geometría y al de la aritmética Boyer (1992).

La función derivada como objeto del cálculo infinitesimal logra su reconocimiento social, científico y matemático en el siglo $X X$, cristalizando el trabajo de muchas personas durante 20 siglos y diferenciándose de otros objetos de las matemáticas como los del álgebra, los de la geometría y los de la aritmética entre otros, Boyer (1992). Según Boyer, en la construcción o descubrimiento del cálculo infinitesimal en su etapa final fue necesario la confrontación y contrastación de los métodos geométricos de Cavallieri y Barrow, con los métodos analíticos de: Descartes, Fermat y Wallis, con los métodos aritméticos de:Roberval, Fermat y Wallis y con los métodos cinemáticos de: Torricelli, Roberval y Barrow. En este sentido es evidente entonces que la influencia de la descendencia histórica de Newton en la cinemática como lo fueron: Arquímedes, Galileo, Torricelli, Roberval y Barrow; así como la influencia de la descendencia histórica de Leibniz en el atomismo, representada por: Demócrito, Kepler, Cavalieri, Fermat, Pascal y Huygens fueron quienes permitieron a Newton y Leibniz el logro del cálculo infinitesimal por caminos diferentes, con lenguajes diferentes también, pero que permitió solucionar los cuatro problemas mencionados para los que la humanidad no había encontrado soluciones hasta entonces.

En el estudio hecho por Boyer, así como en otros se ha evidenciado que en el desarrollo histórico del cálculo primero emergió el proceso de integración, luego lo hizo el proceso de la derivación, posterior a estos el del límite y por último en 1960 el de la función como objeto matemático y el del rigor del lenguaje matemático; mientras que en la Didáctica de la Matemática primero se enseña desde el rigor del lenguaje matemático la función como objeto, luego el límite, la derivada y por último la integral. En este sentido se presentan obstáculos (Epistemológicos, psicológicos y didácticos), Radford (1997) o conflictos semióticos, Godino (2007).

\section{Conclusiones}

$>$ Se evidencia desde la historia de las matemáticas que fueron muchas las personas que a través de distintas épocas ayudaron con sus trabajos a construir lo que hoy conocemos como cálculo diferencial e integral.

$>$ El rigor de las matemáticas como lo conocemos hoy es producto del refinamiento y avance de las matemáticas a través de los trabajos de cientos de matemáticos en distintas épocas.

$>$ El proceso de desarrollo de la función derivada es complejo, demorado y tortuoso. La historia da cuenta que su desarrollo no fue lineal, ni producto de la genialidad de una o dos personas.

$>$ En el proceso histórico de la función derivada subyacen por lo menos tres epistemologías: la de Lagrange (1736-1813), la de Cauchy (1789-1957) y la de Robinson (1918-1974). 
$>$ La Didáctica de la Matemática reporta trabajos e investigaciones en su mayor parte en la epistemología de Cauchy, siendo la más rigurosa y difícil de aprender por parte de estudiantes de último año del colegio o primeros semestres de universidad.

$>$ El lenguaje de la función derivada cambia de acuerdo con la epistemología usada.

\section{Bibliografía}

Acosta, E., Delgado, C. (1994). IFrecht vs Caratheodory", American Mathematical Monthly, Vol. 101, No. 2, 4, April.

ARTIGUE, M., Douady, R., Moreno, L., Gómez, P. (Ed). (1995). Ingeniería didáctica en Educación Matemática, pp 97-140. “Una Empresa Docente" \& Grupo Editorial Iberoamérica. Impreso en México.

Boyer, C. (1992). Historia de la Matemática, versión Española de Mariano Martínez Pérez, Alianza Universidad Textos, Madrid España.

Cantoral, R., Miron, H. (2000). Sobre el estatus de la noción de derivada: de la epistemología de Joseph Louis Lagrange al diseño de una situación didáctica, Revista Latinoamericana de investigación en Matemática Educativa, noviembre, año/vol 3, número 003. Comité Latinoamericano de Matemática Educativa, Distrito Federal, México, pp. 265-292

Keisler, J. (2007). Elementary Calculus, and infinitesimal approach, second Edition, University of Wisconsin. This work is licensed under the Creative Commons. Attribution-NonCommercial-ShareAlike License. To view a copy of this licence, visit http://creativecommons.org/licenses/by-nc-sa/2.0/ or send a letter to Creative Commons, 559 Nathan Abbott Way, Stanford, California, 93405, USA

Caratheodory C., Theory of Functions of a Complex Variable, Chelsea, New

York, 1954.

Fréchet, M. (1925). La Notion de differentielle dans l'analyse generale, C. R. Acad. Sci. (Paris), No. 180.

Kuhn S. (1991). IThe Derivative a la Caratheodory", American Mathematical Monthly, Vol. 98, No. 1, January.

Nicolescu L. J., IOn Some Properties of the direct second order di®erentials in G^ateaux Sense", Review Mathematics Pures Appicated, No. 8 (1963). 